\title{
Erratum to: Effect of meteorological variables on the incidence of lower urinary tract infections
}

\author{
M. E. Falagas • G. Peppas • D. K. Matthaiou • \\ D. E. Karageorgopoulos $・$ N. Karalis $\cdot$ G. Theocharis
}

Published online: 18 September 2009

(C) Springer-Verlag 2009

\section{Erratum to: Eur J Clin Microbiol Infect Dis DOI 10.1007/s10096-008-0679-z}

The Abstract was omitted from the original article.

\begin{abstract}
Several types of infections involving the respiratory tract have a seasonal variation. We further examined whether lower urinary tract infections (UTIs) are associated with meteorological parameters. We retrospectively evaluated the correlation of the weekly percentage of house call visits for lower UTIs (relatively to all house call visits, excluding those for respiratory tract infections), performed by "SOS Doctors" specialized physicians in Attica, Greece
\end{abstract}

The online version of the original article can be found at http://dx.doi. org/10.1007/s10096-008-0679-z.

M. E. Falagas $(\bowtie) \cdot$ G. Peppas $\cdot$ D. K. Matthaiou

D. E. Karageorgopoulos

Alfa Institute of Biomedical Sciences (AIBS),

9 Neapoleos Street, 15123 Marousi,

Athens, Greece

e-mail: m.falagas@aibs.gr

\section{E. Falagas}

Department of Medicine, Henry Dunant Hospital,

Athens, Greece

M. E. Falagas

Deparment of Medicine, Tufts University School of Medicine,

Boston, MA, USA

G. Peppas $\cdot$ G. Theocharis

SOS Doctors,

Athens, Greece

N. Karalis

National Technical University of Athens,

Athens, Greece
(1/11/2000-18/1/2005), with the average weekly temperature and humidity, recorded at the same area, 3 days earlier. Three thousand two hundred and twenty-one visits for lower UTIs were recorded in patients of $62.9 \pm 21.0$ years of age. House call visits for lower UTIs, as defined above, correlated with the average weekly temperature (Spearman's rho +0.468 ) and humidity (Spearman's rho -0.394 ); similarly, if respiratory tract infections were not excluded from the calculations (Spearman's rho +0.491 and -0.406 , respectively); or if a 2-day lag between measurements was used (Spearman's rho +0.468 and -0.386 , respectively). All the above findings were significant $(p<0.001)$. In conclusion, in a population that consisted mainly of patients of advanced age, higher temperature and decreased humidity are associated with an increase in house call visits for lower UTIs. The awareness of this association may facilitate preventive public health strategies. 